\title{
18. Origin and Evolution of Recent Leonid Meteor Showers*
}

\author{
Bruce A. McIntosh \\ National Research Council of Canada \\ Ottawa, Ontario
}

\begin{abstract}
The four most prominent returns of the Leonid shower in the past decade fall into two broad classes. The 1966 and 1969 showers were of short duration, had a high proportion of small particles, and occurred with the longest apparent delay after the perihelion passage of the parent comet Temple-Tuttle. By contrast, the 1961 and 1965 returns were of long duration, and had more large particles. The 1961 return preceded the comet.

There are three major influences on particle orbits: ejection velocity, radiation pressure, and close encounters with planets. The observations are explainable in a qualitative way on the basis of the first two. But some speculation concerning the results of planetary perturbations must be invoked.
\end{abstract}

$\mathrm{R}$ ADAR observations of the Leonid meteor shower during the 13-year period from 1957 to 1969 have been described previously (McIntosh and Millman, 1970; McIntosh, 1970). Detailed examination of these data along with the orbital parameters of the supposed parent comet $\mathrm{P} /$ Temple-Tuttle (1965 IV) (Marsden, 1968) allow some speculation about the evolution of this shower in recent times. Its past history in terms of planetary perturbations has been discussed by Kazimirčak-Polonskaja et al. (1968).

\section{OBSERVATIONAL EVIDENCE}

The four most prominent returns of the shower in the past decade fall into two broad classes. The 1966 and 1969 showers were of short duration, had a high proportion of small particles, and occurred with the longest apparent delay after the perihelion passage of the comet. In contrast,

\footnotetext{
* The author has discovered an error in his calculations that modifies the results as presented at the symposium. This paper is a revised version.
}

the 1961 and 1965 returns were of long duration, and had more large particles. The 1961 return appeared to precede the comet. Quantitative values are shown in table 1 . Here $\Delta T$ is the delay after the comet passed the descending node and $s$ is determined from the assumption of a massdistribution model such that the number of particles having masses between $m$ and $m+d m$ is given by

$$
d N \propto m^{-s} d m
$$

The uncertainty in shower duration for 1965 results from the fact that the radiant was below the horizon at Ottawa near the peak of the shower. Hence the duration to $1 / 4$ strength is the result of a somewhat uncertain extrapolation. For 1961, the uncertainty is due to equipment failure for a period of time.

Figure 1 shows the positions of the comet in its orbit at the times of the returns listed in the table. It is apparent that there are large spacings between the comet and those points along the orbit where there were significant showers.

Figure 2 shows in more detail the geometry as 
TABLE 1.-Observational Data on the Four Major Leonid Showers in the 1960's

\begin{tabular}{|c|c|c|c|}
\hline Year & $\Delta T$ (days) & Mass index $s$ & $\begin{array}{c}\text { Duration to } \\
1 / 4 \text { strength (hr) }\end{array}$ \\
\hline 1961 & -1266 & 1.9 & $(\sim 24)$ \\
\hline 1965 & +195 & 1.6 & $\sim 36$ \\
\hline 1966 & 560 & 2.2 & 1.2 \\
\hline 1969 & +1656 & $\sim 2.4$ & 1.3 \\
\hline
\end{tabular}

the Earth passes through the plane of the comet's orbit. We show the distance and angle from the comet orbit to the Earth in a series of planes perpendicular to the comet's orbit. The planes are two hours of Earth motion apart. The distance of closest approach is $0.003 \mathrm{AU}$ or $4.5 \times 10^{5} \mathrm{~km}$. The 1965,1966 , and 1969 returns are shown in position with respect to the node. The spreading out of the 1965 concentration of particles and the lack of any very definite peak is in distinct contrast to the narrow, sharply peaked concentrations of 1966 and 1969. The reader is reminded that observations of this shower have a finite sampling period with dead-time intervals when the radiant is below the horizon. These are shown by McIntosh and Millman (1970), their figure 2.

\section{ORIGIN OF THE PARTICLE CONCENTRATIONS AND THEIR DIFFERENT CHARACTERISTICS}

There are three major influences on particle orbits: ejection velocity, radiation pressure, and close encounters with planets. According to Whipple's icy conglomerate model for comets, (Whipple, 1951) particles will be ejected from a comet with velocities, $v$, varying as

$$
v \propto r^{-q} / 8\left(b_{\rho}\right)^{-1 / 2}
$$

where $r$ is the distance from the sun

$b$ is particle radius

and $\rho$ its density.

Particles are presumed to be emitted initially on the surface facing the Sun but their final motion is complicated by two factors: possible rotation of the comet and the fact that the final motion of the escaping gases, which provide the momentum

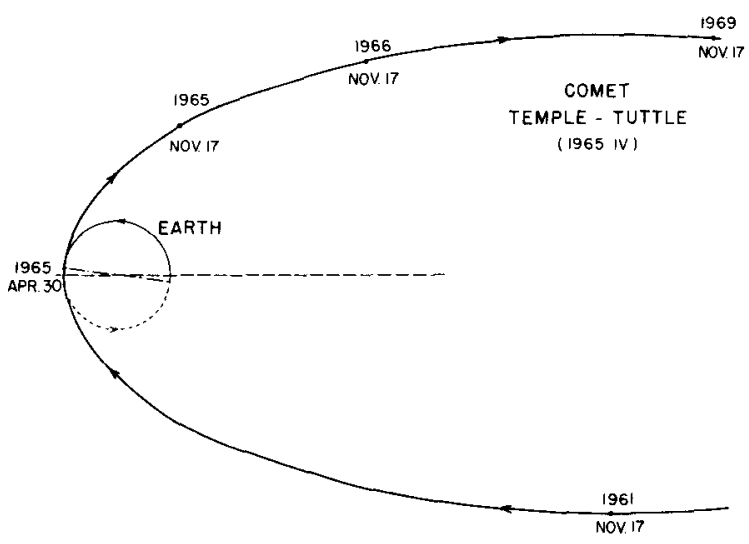

Figure 1.-Positions of the comet $P /$ Temple-Tuttle (1965 IV) at the times when the Earth encountered major concentrations of Leonid meteors in the 1960's. Comet perihelion was April 30, 1965, and the line of nodes is $7.4^{\circ}$ from the major axis.

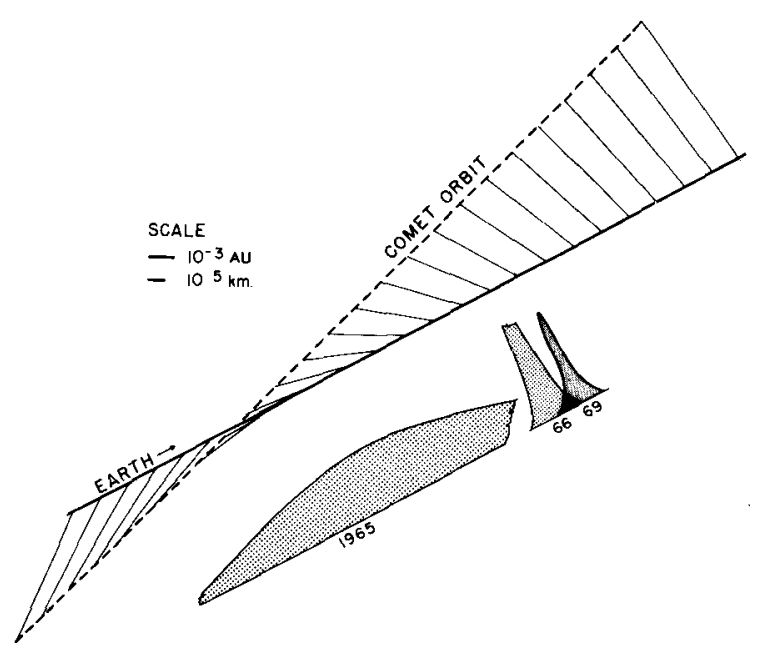

Figure 2.-Detail of the Earth passing close to the comet's orbit. Lines from comet orbit to Earth orbit are at 2-hr intervals of the Earth's motion and represent angles and distances in planes perpendicular to the comet's orbit. Rate curves are in correct position with respect to the node but are otherwise only pictorial.

transfer for carrying off the particles, is predominantly in the antisolar direction. Figure 3 indicates some of the parameters necessary for a quantitative discussion of the effect of ejection velocity on orbital parameters. Particles are ejected at a position where the radius vector is $r$ and makes an angle $\nu$ with perihelion. Ejection velocity is resolved into components $v_{r}$ in the 
radial direction and $v_{b}$ perpendicular to the radius in the plane of the orbit.

Radiation pressure is usually taken into account by expressing it as a fraction, $\beta$ say, of the gravitational force. The orbital speed $V$ of the particles is then given by

$$
V^{2}=\mu(1-\beta)\left(\frac{2}{r}-\frac{1}{a}\right)
$$

where $\mu$ is the solar gravitational constant and $a$ the semimajor axis. The ratio $\beta$ depends on particle size as,

$$
\beta \propto \frac{1}{\rho b}
$$

It is now of interest to determine when the particle will again reach the node and what the value of the radius vector will be. These parameters may be assessed by determining the perturbations to the original orbit. The delay with which the particle follows the comet is determined by the change in orbital period resulting from the change in semi-major axis, $a$. For the radius vector, since the nodal passage is within a few degrees of perihelion, it is sufficient to consider changes in the perihelion distance $q$. These perturbations (exclusive of any planetary effects) are

$$
\begin{aligned}
\frac{d a}{a}=\frac{1}{1-e}\left[\beta \frac{1+e^{2}+2 e \cos \nu_{0}}{1+e}\right. & \\
& \left.+2 e \sin \nu_{0} \frac{v_{r}}{V_{q}}+\left(1+e \cos \nu_{0}\right) \frac{v_{b}}{V_{q}}\right]
\end{aligned}
$$$$
\frac{d q}{q}=\beta \frac{1-\cos \nu_{0}}{1+e}-\sin \nu_{0} \frac{v_{r}}{V_{q}}
$$

$$
+\frac{\left(1-\cos \nu_{0}\right)\left(2+e+e \cos \nu_{0}\right)}{1+e \cos \nu_{0}} \frac{v_{b}}{V_{q}}
$$

where $e$ is the eccentricity and $V_{q}$ is the comet velocity at perihelion. Note that the perturbation in semi-major axis is strongly influenced by the factor $1 /(1-e)$ which is about 10 in the case of the Leonids. The magnitude of the perturbations is illustrated by the values in table 2 which have been calculated for a comet of $1 \mathrm{~km}$ radius at $1 \mathrm{AU}$ and particles of density $1 \mathrm{~g} / \mathrm{cm}^{3}$. It is apparent that the perturbations due to radiation pressure are of roughly equal magnitude to those due to ejection velocity.

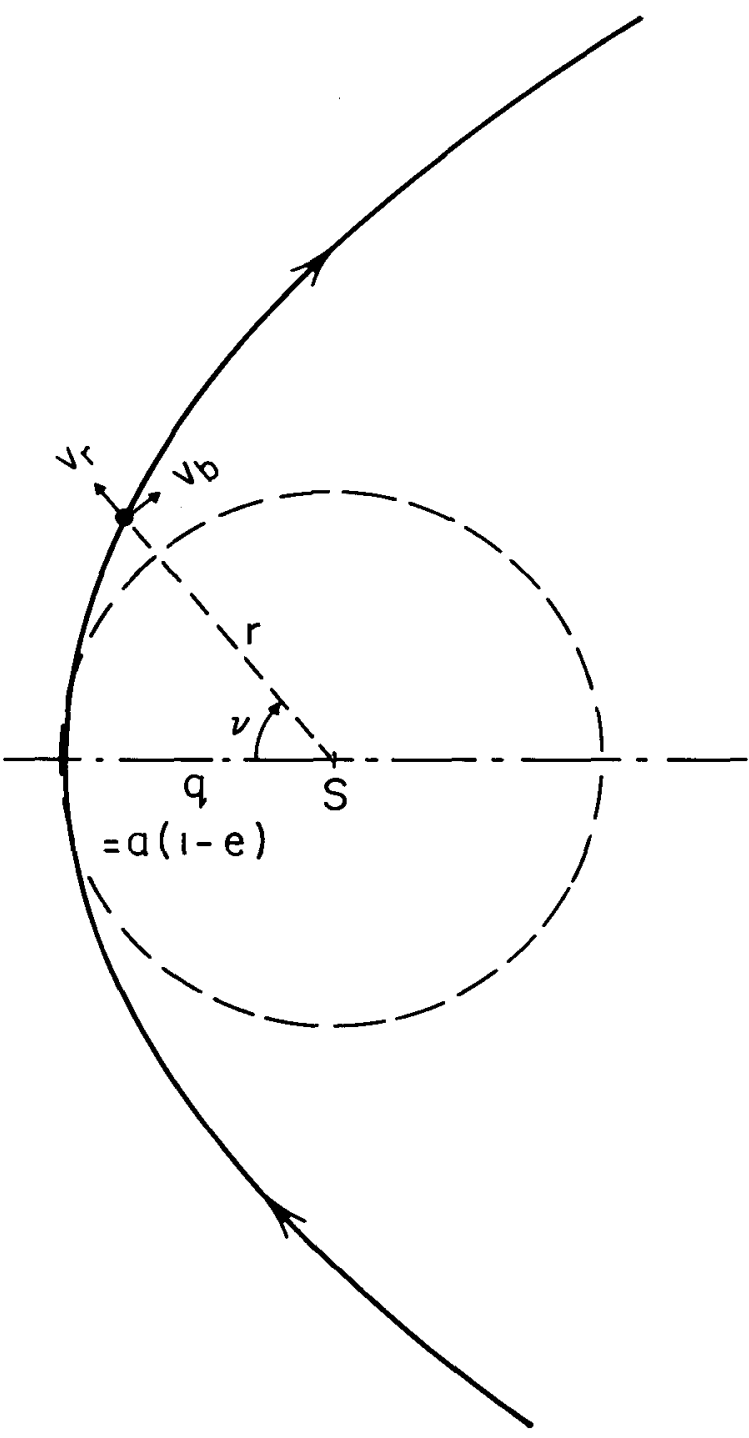

Figure 3.-Necessary parameters for considering ejection from the comet of particles with velocities $v_{r}, v_{b}$.

Calculations have been made on a model with particles of density $1 \mathrm{~g} / \mathrm{cm}^{3}$ ejected in four directions $\pm v_{r}, \pm v_{b}$. Release of particles from the comet has been followed from $r=2$ AU through perihelion and out to $2 \mathrm{AU}$. Particles in three mass classes at $1 \mathrm{~g}, 0.1 \mathrm{~g}$, and $0.01 \mathrm{~g}$ are emitted in numbers and velocities according to the foregoing theory.

The resultant positions of the particles in terms of $\Delta q$ and $\Delta T$ are shown in figure 4. The values of delay are for one period of revolution of the comet. The distributions for each particle 
TABLE 2.-Typical values of ejection velocity and radiation pressure parameter for particles of density $1 \mathrm{~g} / \mathrm{cm}^{3}$ released from a comet of radius $1 \mathrm{~km}$ at $1 \mathrm{AU}$

\begin{tabular}{|c|c|c|c|}
\hline \multirow{2}{*}{$\begin{array}{c}\text { Particle } \\
\text { mass }\end{array}$} & \multicolumn{2}{|c|}{ Ejection } & \multirow{2}{*}{$\underset{\beta}{\text { Radiation }}$} \\
\hline & $v$ & $v / V_{q}$ & \\
\hline $1 \mathrm{~g}$ & $8 \mathrm{~m} / \mathrm{s}$ & $2 \times 10^{-4}$ & $1 \times 10^{-4}$ \\
\hline $0.01 \mathrm{~g}$ & $18 \mathrm{~m} / \mathrm{s}$ & $4.5 \times 10^{-4}$ & $4 \times 10^{-4}$ \\
\hline
\end{tabular}

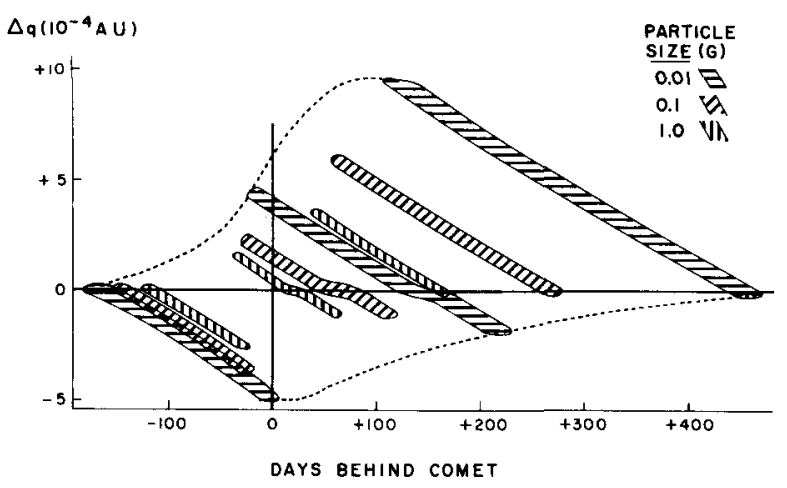

Figure 4.-Perturbed position of particles after one orbit for ejection in four directions $\left( \pm v_{r}, \pm v_{b}\right)$, for three particle size classes. The dotted outline encloses the region of $\Delta q, \Delta T$ values for isotropic ejection of 0.01-g particles.

size class are essentially similar in form. In each case there are three branches, the outer two resulting from ejection in the $( \pm)$ direction of the orbital motion. The one with the greatest delay and lying outside the original orbit results from the $+v_{b}$ component, and the branch preceding the comet and inside the orbit is associated with $-v_{b}$. The central branch which has a kink where it crosses the orbit results from ejection in the plus or minus radial direction. Either direction $\left( \pm v_{r}\right)$ exclusively will produce this branch because of the change of sign of the $\sin \nu$ factor.

If particles are emitted isotopically, the distribution in $\Delta q, \Delta T$ becomes that shown by the dotted outline in figure 4 , for $0.01 \mathrm{~g}$ particles. There will be a further smearing of this distribution in the orbital plane since in reality there will be a range of particle sizes with a distribution of velocities in each size class. Thus there will be a smeared-out, thin plane of particles, the larger ones tending to be more concentrated close to the comet while the smaller ones will achieve positions mostly outside the original orbit and very quickly lagging behind the comet. The thinness of the layer becomes apparent when one notes that in 1966 and 1969 the width to $1 / 4$ strength was about 3 Earth diameters. There were significant concentration changes in distances less than one Earth diameter. It is possible that this effect is due to concentrations in the direction within the orbital plane, but because of the rapid spreading of particles in the plane it seems more plausible that the concentration is that of a thin plane.

The distance of closest approach between the Earth and the comet orbit at this recent passage was $0.003 \mathrm{AU}$ some 3 times the maximum $\Delta q$ perturbation indicated in figure 4 . Only very small particles could have achieved orbits at this distance for the uniform ejection model described here. From this point on then, one must speculate as to the mechanism which allows particle orbits at this distance from the comet orbit. Irregular ejection at higher velocities-an explosion-type of event, is a possibility. One can retain the uniform ejection model by noting that planetary encounters perturb the perihelion distance (see fig. 2 of Kazimirčak-Polonskaja et al., 1968). Once the particles begin to diverge from the comet, gravitational perturbations will be different for the particles than for the comet itself. This is essentially the problem that the Russian authors have attempted, that of following a particular swarm of particles through the gravitational perturbations of many revolutions. The results are only as good as the initial conditions which in most cases are not known to sufficient precision.

Thus, assuming that the particles have been moved out to an Earth-encounter position by planetary perturbations, we can examine figure 4 in the light of the observations in table 1 . The 1969 return consisted mainly of smaller particles at a delay of 1600 days behind the comet. If we place greatest emphasis on particles emitted close to perihelion, figure 4 indicates a delay of 200 to 400 days per revolution, for small particles only $(0.01 \mathrm{~g})$. These particles were probably ejected about five revolutions ago or are between 100 and 200 years old.

Moving back toward the comet in figure 4, one finds particles of all three size classes at a delay of about 100 days per orbit. Hence for the return of 
1966 at a total delay of 560 days, an age of five or six orbital revolutions is again indicated. There arises now the interesting question: Are the 1966 and 1969 returns associated with the same ejection event?

If one visualizes the form of figure 4 going over to a continuous distribution of particle sizes and with a distribution of velocities for each size increment, it seems doubtful that there will remain concentrations sufficient to explain the high rates of 1966 without there being significantly high rates in 1967 and 1968. This question cannot be resolved with our own data, since at Ottawa the radiant was below the horizon at the time of passage through the orbital plane in 1968 and was poorly placed in 1967. Rate information for these years is lacking. It is, of course, possible that the particles observed in 1966 resulted from a large outburst at a particular point on the orbit.

In 1965 , the shower width to $1 / 4$ strength was about 36 hours with indication of some activity over 4 days. This indicates more than an order of magnitude increase in the thickness of the particle belt as compared with 1966 and 1969. As well as this difference, we note that the 1965 return comprised mostly large particles.

Kazimirčak-Polonskaja et al. (1968) have shown that the longitude of the node has undergone large perturbations, particularly due to close approaches to Jupiter (see also Guth, 1968). However, the spread in nodal perturbations as indicated by the width of the 1965 return is of the same order as the total shift over the past 200 years. Thus the spread in the 1965 return must have resulted from planetary perturbations over many centuries. The unusual feature of the 1965 event is that although probably the oldest, it was closest to the present position of the comet with $\Delta T$ of only 195 days. A glance at figure 4 shows two possible explanations. There is the possibility of very small delays, a few days per revolution, leading to a very indefinite age of perhaps 20 to 100 revolutions. The alternative is to choose the greater interval of one of the outer branches, say \pm 100 days, and allow it to exist for so long that the total delay or advance is an entire period. This requires some 150 to 200 revolutions or 6000 years roughly. The 1961 shower was closer in nature to the 1965 event than to the others, having roughly the same duration but a slightly higher content of intermediate sized particles. This may represent either an advance of 1266 days or a delay of one period minus 1266 days.

\section{PROBLEMS AND CONCLUSIONS}

The difference in age between the two basic types of shower seems a necessary conclusion. To put actual values on the ages is more difficult. That the particles very quickly become distributed around the orbit is apparent from figure 4. One would expect that in the older showers, such as the 1965 return, the Earth would encounter fewer particles, because of the dispersion along the orbit. From the observational evidence (McIntosh and Millman, 1970) the Earth encountered as many large particles (echoes $\geq 1$ s duration) in 1965 as in the shower of 1966 . The ages of the showers are in the ratio of about $30: 1$. If the original concentrations were the same (there is in fact no reason why this should be so) the particle density along the orbit should be reduced by $1 / 30$ in the 1965 return. But because of the nodal broadening, the Earth is immersed in the stream some 30 times longer in 1965 than in 1966 and is therefore sampling the same integrated concentration in both cases.

One of the major problems with the evolution of the Leonids is whether ejection of particles from the comet has taken place uniformly as the comet swings around the Sun, or whether only as discrete ejection at a single position along the orbit. Figure 4 indicates that uniform ejection would form a wide belt which in a few tens of revolutions would be spread around the orbit. The lack of any degree of shower activity except within a few years of perihelion passage argues against uniform ejection at repeated passages. 


\section{REFERENCES}

GUTн, V., 1968. Perturbations of the line of nodes of the Leonids during the years 1866-1966, in Physics and Dynamics of Meteors, edited by L̆. Kresák and P. M. Millman, D. Reidel Publ. Co., Dordrecht, Holland, 476-480.

Kazimirčak-Polonskaja, E. I., Beljaev, N. A., Astapovič, I. S., and Terenteva, A. K., 1968. Investigation of perturbed motion of the Leonid meteor stream, in Physics and Dynamics of Meteors, edited by L. Kresák and P. M. Millman, D. Reidel Publ. Co., Dordrecht, Holland, 449-475.

Marsden, B. G., 1968. Reports on the progress of astronomy, Comets, Quart. J. Roy. Astron. Soc., 9, 304-321.

McIntosh, B. A., 1970. Radar observations of the 1969 Leonid meteor shower, Bull. Radio and Elect. Eng. Div., N.R.C. Can., 20, No. 2, 30-33.

McIntosh, B. A., AND Millman, P. M., 1970. The Leonids by radar-1957 to 1968, Meteoritics, $5,1-18$.

Whipple, F. L., 1951. A comet model. II. Physical relations for comets and meteors, Astrophys. $J ., 113,464-474$. 